\title{
AdAPTIVe Controller Design FOR THE ANTI-SYNCHRONIZATION OF HYPERCHAOTIC YANG AND Hyperchaotic PANg Systems
}

\author{
Sundarapandian Vaidyanathan ${ }^{1}$ \\ ${ }^{1}$ Research and Development Centre, Vel Tech Dr. RR \& Dr. SR Technical University \\ Avadi, Chennai-600 062, Tamil Nadu, INDIA \\ sundarvtu@gmail.com
}

\begin{abstract}
In the anti-synchronization of chaotic systems, a pair of chaotic systems called drive and responsesystems are considered, and the design goal is to drive the sum of their respective states to zero asymptotically. This paper derives new results for the anti-synchronization of hyperchaotic Yang system (2009) and hyperchaotic Pang system (2011) with uncertain parameters via adaptive control. Hyperchaotic systems are nonlinear chaotic systems withtwo or more positive Lyapunov exponents and they have applications in areas like neural networks, encryption, secure data transmission and communication. The main results derived in this paper are illustrated with MATLAB simulations.
\end{abstract}

\section{KEYWORDS}

Hyperchaos, Adaptive Control, Anti-Synchronization, Hyperchaotic Systems.

\section{INTRODUCTION}

Since the discovery of a hyperchaotic system by O.E.Rössler ([1], 1979), hyperchaotic systems are known to have characteristics like high security, high capacity and high efficiency. Hyperchaotic systems are chaotic systems having two or morepositive Lyapunov exponents. They are applicable in several areas like oscillators [2], neural networks [3], secure communication [45], data encryption [6], chaos synchronization [7], etc.

The synchronization problem deals with a pair of chaotic systems called the drive and response chaotic systems, where the design goal is to drive the difference of their respective states to zero asymptotically [8-9].

The anti-synchronization problem deals with a pair of chaotic systems called the drive and response systems, where the design goal is to drive the sum of their respective states to zero asymptotically.

The problems of synchronization and anti-synchronization of chaotic and hyperchaotic systems have been studied via several methods like active control method [10-12], adaptive control method [13-15],backstepping method [16-19], sliding control method [20-22] etc.

This paper derives new results for the adaptive controller design for the anti-synchronization of hyperchaotic Yang systems ([23], 2009) and hyperchaotic Pang systems ([24], 2008) with 
unknown parameters. The main results derived in this paper were proved using adaptive control theory [25] and Lyapunov stability theory [26].

\section{Problem Statement}

The drive system is described by the chaotic dynamics

$$
\dot{x}=A x+f(x)
$$

where $A$ is the $n \times n$ matrix of the system parameters and $f: R^{n} \rightarrow R^{n}$ is the nonlinear part.

The response system is described by the chaotic dynamics

$$
\dot{y}=B y+g(y)+u
$$

where $B$ is the $n \times n$ matrix of the system parameters, $g: R^{n} \rightarrow R^{n}$ is the nonlinear part and $u \in R^{n}$ is the active controller to be designed.

For the pair of chaotic systems (1) and (2), the design goal of the anti-synchronization problemis to construct a feedback controller $u$, which anti-synchronizes their states for all $x(0), y(0) \in R^{n}$.

Theanti-synchronization erroris defined as

$$
e=y+x
$$

Theerror dynamics is obtained as

$$
\dot{e}=B y+A x+g(y)+f(x)+u
$$

The design goal is to find a feedback controller $\boldsymbol{u}$ so that

$$
\lim _{t \rightarrow \infty}\|e(t)\|=0 \text { for all } e(0) \in R^{n}
$$

Using the matrix method, we consider a candidate Lyapunov function

$$
V(e)=e^{T} P e,
$$

where $P$ is a positive definite matrix.

It is noted that $V: R^{n} \rightarrow R$ is a positive definite function.

If we find a feedback controller $\boldsymbol{u}$ so that

$$
\dot{V}(e)=-e^{T} Q e
$$

where $Q$ is a positive definite matrix, then $\dot{V}: R^{n} \rightarrow R$ is a negative definite function.

Thus, by Lyapunov stability theory [26], the error dynamics (4) is globally exponentially stable. 
When the system parameters in (1) and (2) are unknown, we need to construct a parameter update law for determining the estimates of the unknown parameters.

\section{HYPERCHAOTIC SYSTEMS}

The hyperchaotic Yang system ([23], 2009) is given by

$$
\begin{aligned}
& \dot{x}_{1}=a\left(x_{2}-x_{1}\right) \\
& \dot{x}_{2}=c x_{1}-x_{1} x_{3}+x_{4} \\
& \dot{x}_{3}=-b x_{3}+x_{1} x_{2} \\
& \dot{x}_{4}=-d x_{1}-\varepsilon x_{2}
\end{aligned}
$$

where $a, b, c, d$ are constant, positive parameters of the system.

The Yang system (8) exhibits a hyperchaotic attractor for the parametric values

$$
a=35, \quad b=3, c=35, d=2, \varepsilon=7.5
$$

The Lyapunov exponents of the system (8) for the parametric values in (9) are

$$
\lambda_{1}=0.2747, \quad \lambda_{2}=0.1374, \quad \lambda_{3}=0, \quad \lambda_{4}=-38.4117
$$

Since there are two positive Lyapunov exponents in (10), the Yang system (8) is hyperchaotic for the parametric values (9).

The phase portrait of the hyperchaotic Yang system is described in Figure 1.

The hyperchaotic Pang system ([24], 2011) is given by

$$
\begin{aligned}
& \dot{x}_{1}=\alpha\left(x_{2}-x_{1}\right) \\
& \dot{x}_{2}=\gamma x_{2}-x_{1} x_{3}+x_{4} \\
& \dot{x}_{3}=-\beta x_{3}+x_{1} x_{2} \\
& \dot{x}_{4}=-\delta\left(x_{1}+x_{2}\right)
\end{aligned}
$$

where $\alpha, \beta, \gamma, \delta$ are constant, positive parameters of the system.

The Pang system (11) exhibits a hyperchaotic attractor for the parametric values

$$
\alpha=36, \quad \beta=3, \gamma=20, \quad \delta=2
$$

The Lyapunov exponents of the system (9) for the parametric values in (12) are

$$
\lambda_{1}=1.4106, \quad \lambda_{2}=0.1232, \quad \lambda_{3}=0, \quad \lambda_{4}=-20.5339
$$

Since there are two positive Lyapunov exponents in (13), the Pang system (11) is hyperchaotic for the parametric values (12).

The phase portrait of the hyperchaotic Pang system is described in Figure 2. 

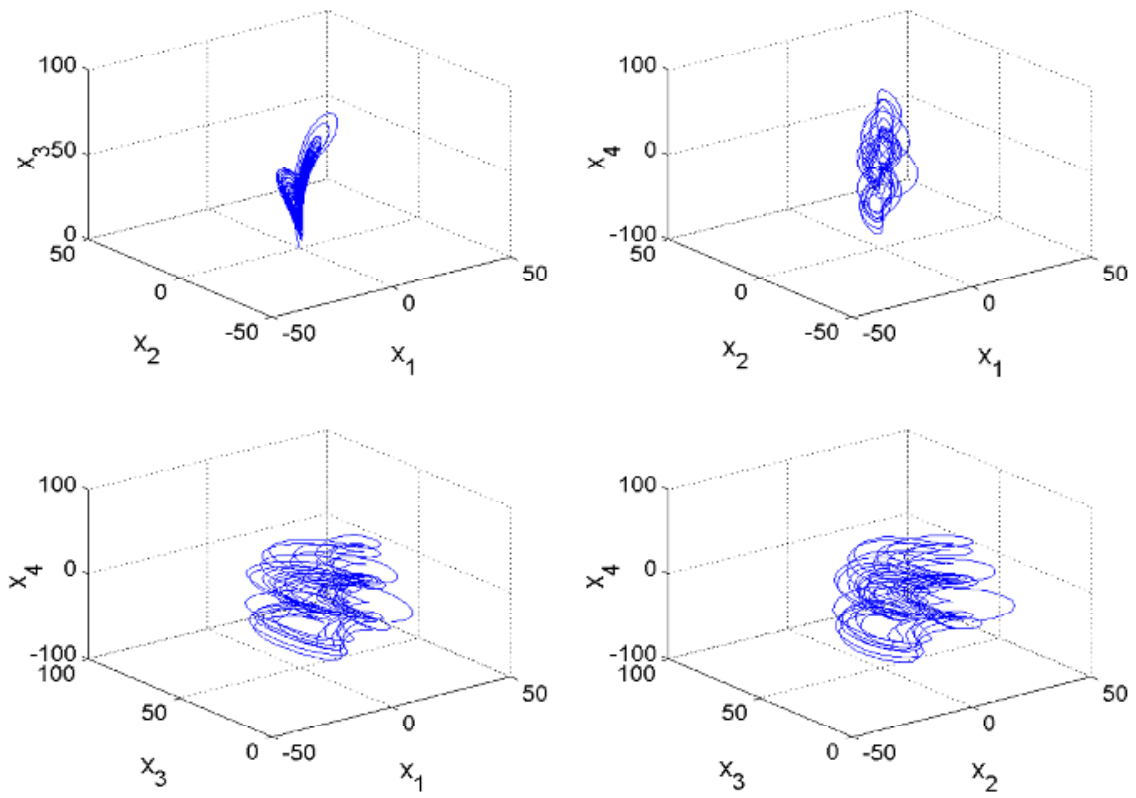

Figure 1. The Phase Portrait of the Hyperchaotic Yang System
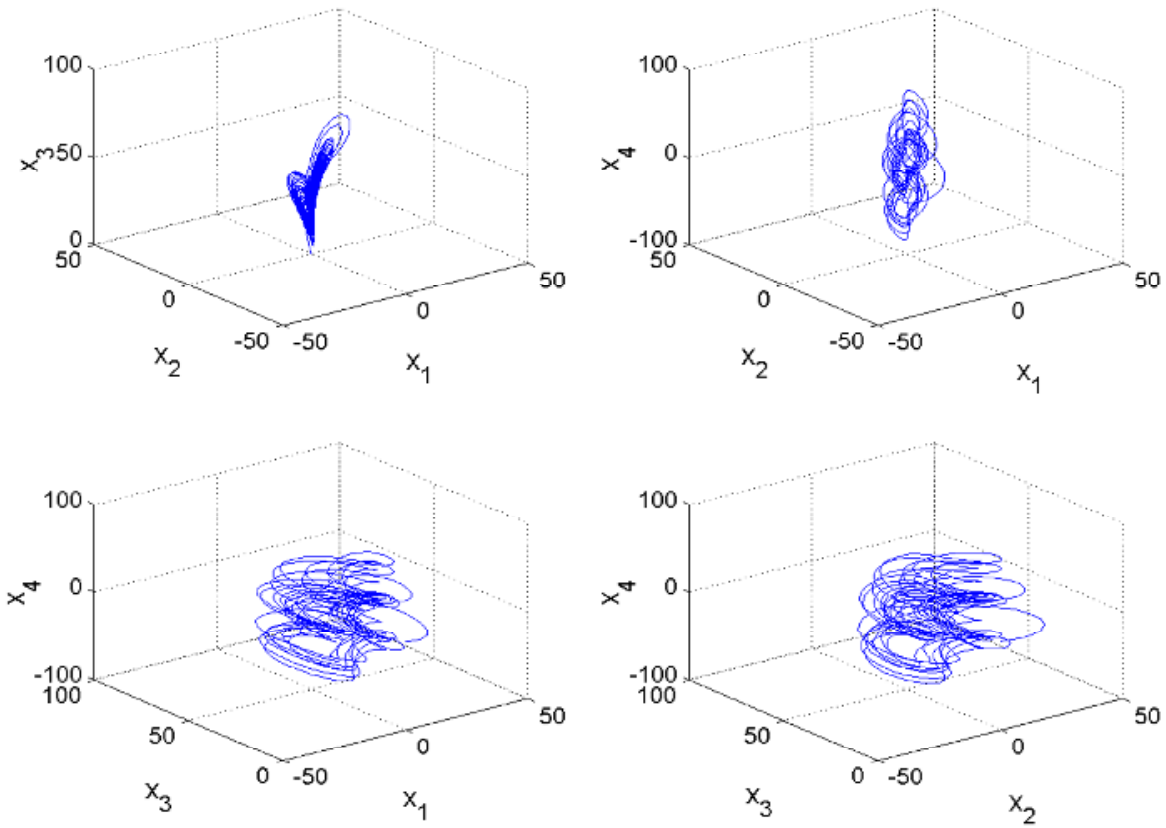

Figure 2. The Phase Portrait of the Hyperchaotic Pang System 


\section{Adaptive Control Design for the Anti-Synchronization of HYPERCHAOTIC YANG SYSTEMS}

In this section, we design an adaptive controller for the anti-synchronization of two identical hyperchaotic Yang systems (2009) with unknown parameters.

Thedrive system is the hyperchaotic Yangdynamicsgiven by

$$
\begin{aligned}
& \dot{x}_{1}=a\left(x_{2}-x_{1}\right) \\
& \dot{x}_{2}=c x_{1}-x_{1} x_{3}+x_{4} \\
& \dot{x}_{3}=-b x_{3}+x_{1} x_{2} \\
& \dot{x}_{4}=-d x_{1}-\varepsilon x_{2}
\end{aligned}
$$

where $a, b, c, d, \varepsilon$ are unknown parameters of the system and $x \in R^{4}$ is the state.

The response system is the controlled hyperchaotic Yangdynamics given by

$$
\begin{aligned}
& \dot{y}_{1}=a\left(y_{2}-y_{1}\right)+u_{1} \\
& \dot{y}_{2}=c y_{1}-y_{1} y_{3}+y_{4}+u_{2} \\
& \dot{y}_{3}=-b y_{3}+y_{1} y_{2}+u_{3} \\
& \dot{y}_{4}=-d y_{1}-\varepsilon y_{2}+u_{4}
\end{aligned}
$$

where $y \in R^{4}$ is the state and $u_{1}, u_{2}, u_{3}, u_{4}$ are the adaptivecontrollers to be designed.

For the anti-synchronization, the error $e$ is defined as

$$
e_{1}=y_{1}+x_{1}, \quad e_{2}=y_{2}+x_{2}, \quad e_{3}=y_{3}+x_{3}, \quad e_{4}=y_{4}+x_{4}
$$

Then we derive the error dynamics as

$$
\begin{aligned}
& \dot{e}_{1}=a\left(e_{2}-e_{1}\right)+u_{1} \\
& \dot{e}_{2}=c e_{1}+e_{4}-y_{1} y_{3}-x_{1} x_{3}+u_{2} \\
& \dot{e}_{3}=-b e_{3}+y_{1} y_{2}+x_{1} x_{2}+u_{3} \\
& \dot{e}_{4}=-d e_{1}-\varepsilon e_{2}+u_{4}
\end{aligned}
$$

The adaptive controller to achieve anti-synchronization is chosen as

$$
\begin{aligned}
& u_{1}(t)=-\hat{a}(t)\left(e_{2}-e_{1}\right)-k_{1} e_{1} \\
& u_{2}(t)=-\hat{c}(t) e_{1}-e_{4}+y_{1} y_{3}+x_{1} x_{3}-k_{2} e_{2} \\
& u_{3}(t)=\hat{b}(t) e_{3}-y_{1} y_{2}-x_{1} x_{2}-k_{3} e_{3} \\
& u_{4}(t)=\hat{d}(t) e_{1}+\hat{\varepsilon}(t) e_{2}-k_{4} e_{4}
\end{aligned}
$$


In Eq. (18), $k_{i},(i=1,2,3,4)$ are positive gains and $\hat{a}(t), \hat{b}(t), \hat{c}(t), \hat{d}(t), \hat{\varepsilon}(t)$ are estimates for the unknown parameters $a, b, c, d, \varepsilon$ respectively.

By the substitution of (18) into (17), the error dynamics is simplified as

$$
\begin{aligned}
& \dot{e}_{1}=(a-\hat{a}(t))\left(e_{2}-e_{1}\right)-k_{1} e_{1} \\
& \dot{e}_{2}=(c-\hat{c}(t)) e_{1}-k_{2} e_{2} \\
& \dot{e}_{3}=-(b-\hat{b}(t)) e_{3}-k_{3} e_{3} \\
& \dot{e}_{4}=-(d-\hat{d}(t)) e_{1}-(\varepsilon-\hat{\varepsilon}(t)) e_{2}-k_{4} e_{4}
\end{aligned}
$$

As a next step, we define the parameter estimation errors as

$$
e_{a}(t)=a-\hat{a}(t), e_{b}(t)=b-\hat{b}(t), e_{c}(t)=c-\hat{c}(t), e_{d}(t)=d-\hat{d}(t), e_{\varepsilon}(t)=\varepsilon-\hat{\varepsilon}(t)
$$

Upon differentiation, we get

$$
\dot{e}_{a}(t)=-\dot{\hat{a}}(t), \dot{e}_{b}(t)=-\dot{\hat{b}}(t), \dot{e}_{c}(t)=-\dot{\hat{c}}(t), \dot{e}_{d}(t)=-\dot{\hat{d}}(t), \dot{e}_{\varepsilon}(t)=-\dot{\hat{\varepsilon}}(t)
$$

Substituting (20) into the error dynamics (19), we obtain

$$
\begin{aligned}
& \dot{e}_{1}=e_{a}\left(e_{2}-e_{1}\right)-k_{1} e_{1} \\
& \dot{e}_{2}=e_{c} e_{1}-k_{2} e_{2} \\
& \dot{e}_{3}=-e_{b} e_{3}-k_{3} e_{3} \\
& \dot{e}_{4}=-e_{d} e_{1}-e_{\varepsilon} e_{2}-k_{4} e_{4}
\end{aligned}
$$

We consider the candidate Lyapunov function

$$
V=\frac{1}{2}\left(e_{1}^{2}+e_{2}^{2}+e_{3}^{2}+e_{4}^{2}+e_{a}^{2}+e_{b}^{2}+e_{c}^{2}+e_{d}^{2}+e_{\varepsilon}^{2}\right)
$$

Differentiating (23) along the dynamics (21) and (22), we obtain

$$
\begin{aligned}
\dot{V}= & -k_{1} e_{1}^{2}-k_{2} e_{2}^{2}-k_{3} e_{3}^{2}-k_{4} e_{4}^{2}+e_{a}\left[e_{1}\left(e_{2}-e_{1}\right)-\dot{\hat{a}}\right]+e_{b}\left(-e_{3}^{2}-\dot{\hat{b}}\right) \\
& +e_{c}\left(e_{1} e_{2}-\dot{\hat{c}}\right)+e_{d}\left(-e_{1} e_{4}-\dot{\hat{d}}\right)+e_{\varepsilon}\left(-e_{2} e_{4}-\dot{\hat{\varepsilon}}\right)
\end{aligned}
$$

In view of (24), we choose the following parameter update law:

$$
\begin{array}{ll}
\dot{\hat{a}}=e_{1}\left(e_{2}-e_{1}\right)+k_{5} e_{a}, & \dot{\hat{d}}=-e_{1} e_{4}+k_{8} e_{d} \\
\dot{\hat{b}}=-e_{3}^{2}+k_{6} e_{b}, & \dot{\hat{\varepsilon}}=-e_{2} e_{4}+k_{9} e_{\varepsilon} \\
\dot{\hat{c}}=e_{1} e_{2}+k_{7} e_{c} &
\end{array}
$$

Next, we prove the following main result of this section. 
Theorem 4.1 The adaptive control law defined by Eq. (18) along with the parameter update law defined by Eq. (25) achieveglobal and exponential anti-synchronization of the identical hyperchaotic Yang systems (14) and (15)with unknown parameters for all initial conditions $x(0), y(0) \in R^{4}$. Moreover, the parameter estimation errors $e_{a}(t), e_{b}(t), e_{c}(t), e_{d}(t), e_{\varepsilon}(t)$ globally and exponentially converge to zero for all initial conditions.

Proof.The proof is via Lyapunov stability theory [26] by taking $V$ defined by Eq. (23) as the candidate Lyapunov function. Substituting the parameter update law (25) into (24), we get

$$
\dot{V}(e)=-k_{1} e_{1}^{2}-k_{2} e_{2}^{2}-k_{3} e_{3}^{2}-k_{4} e_{4}^{2}-k_{5} e_{a}^{2}-k_{6} e_{b}^{2}-k_{7} e_{c}^{2}-k_{8} e_{d}^{2}-k_{9} e_{\varepsilon}^{2}
$$

which is a negative definite function on $R^{9}$. This completes the proof.

Next, we illustrate our adaptive anti-synchronization results with MATLAB simulations.The classical fourth order Runge-Kutta method with time-step $h=10^{-8}$ has been used to solve the hyperchaotic Yang systems (14) and (15) with the nonlinear controller defined by (18).

The feedback gains in the adaptive controller (18) are taken as $k_{i}=4,(i=1, \ldots, 9)$.

The parameters of the hyperchaotic Yang systems are taken as in the hyperchaotic case, i.e.

$$
a=35, \quad b=3, c=35, d=2, \varepsilon=7.5
$$

For simulations, the initial conditions of the drive system (14) are taken as

$$
x_{1}(0)=7, \quad x_{2}(0)=16, \quad x_{3}(0)=-23, \quad x_{4}(0)=-5
$$

Also, the initial conditions of the response system (15) are taken as

$$
y_{1}(0)=34, \quad y_{2}(0)=-8, \quad y_{3}(0)=28, \quad y_{4}(0)=-20
$$

Also, the initial conditions of the parameter estimates are taken as

$$
\hat{a}(0)=12, \quad \hat{b}(0)=8, \quad \hat{c}(0)=-7, \quad \hat{d}(0)=5, \quad \hat{\varepsilon}(0)=4
$$

Figure 3 depicts the anti-synchronization of the identical hyperchaotic Yang systems. Figure 4 depicts the time-history of the anti-synchronization errors $e_{1}, e_{2}, e_{3}, e_{4}$.

Figure 5 depicts the time-history of the parameter estimation errors $e_{a}, e_{b}, e_{c}, e_{d}, e_{\varepsilon}$. 

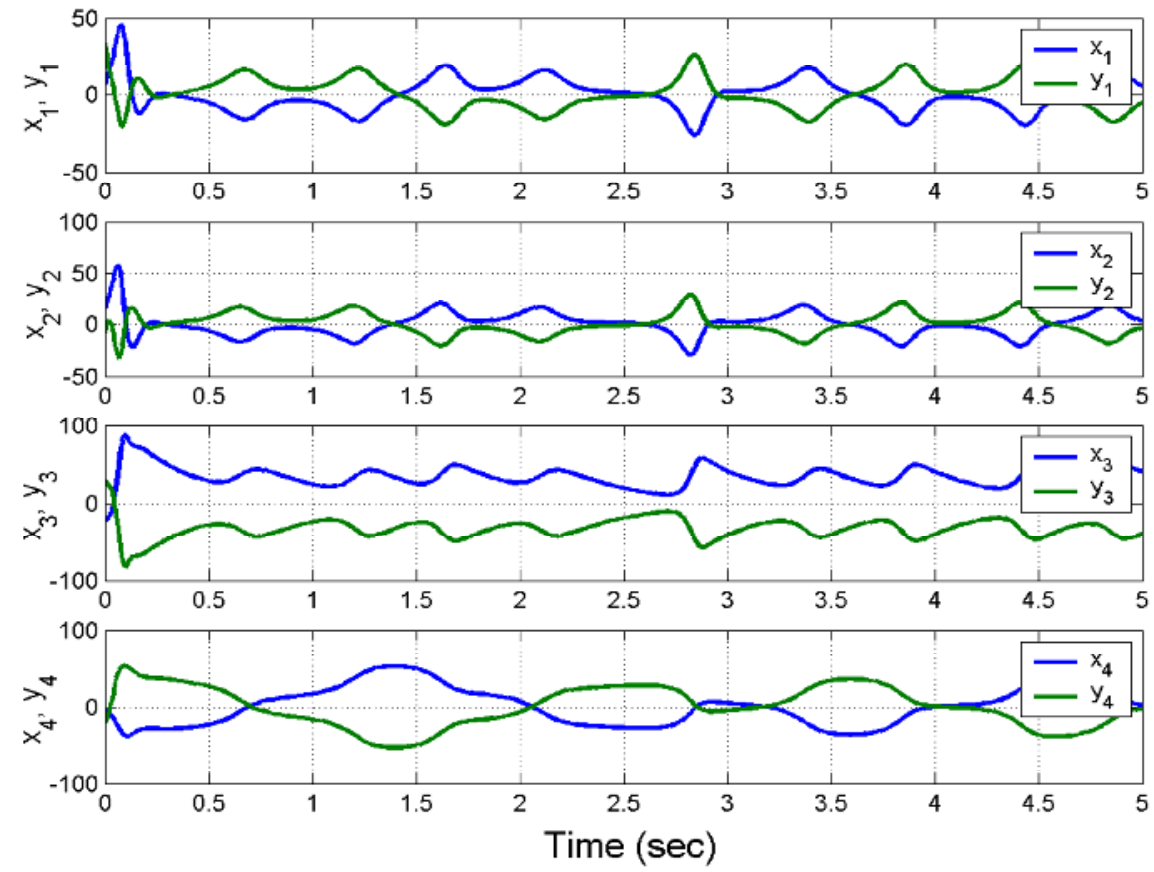

Figure 3. Anti-Synchronization of Identical Hyperchaotic Yang Systems

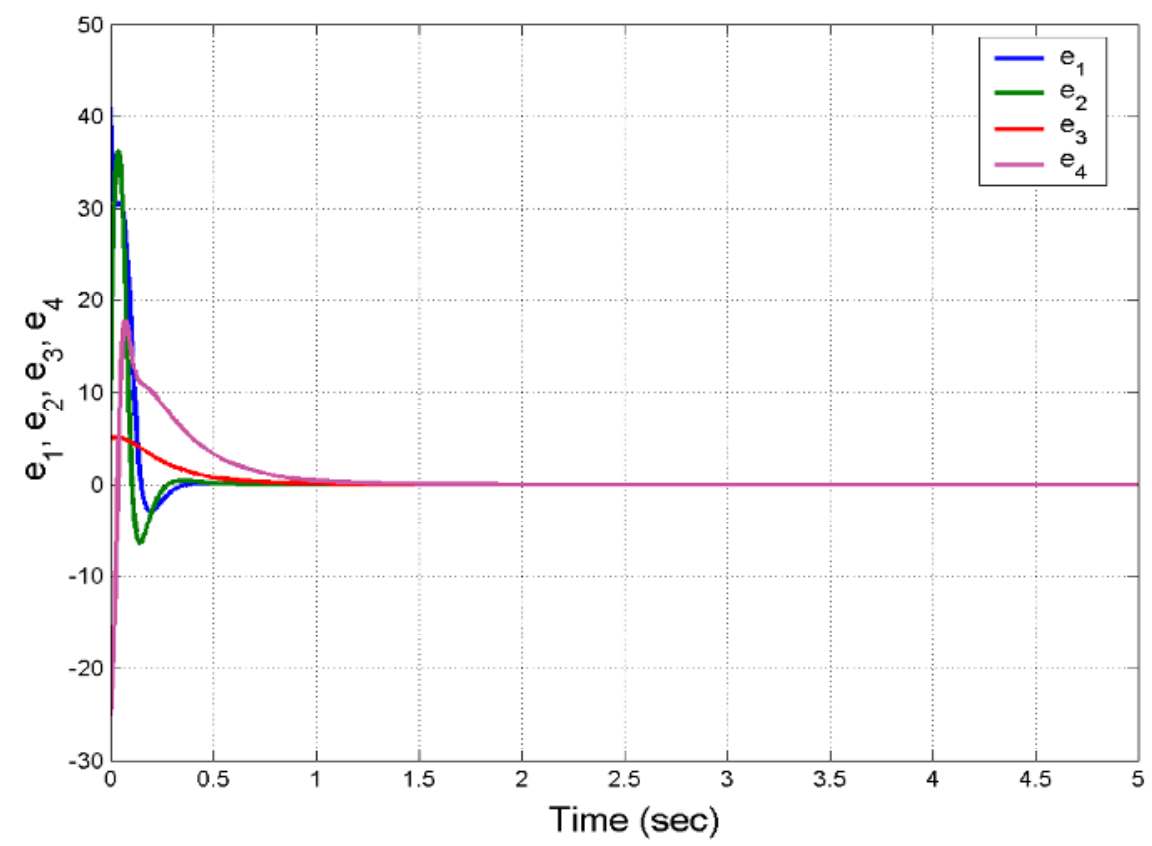

Figure 4. Time-History of the Anti-Synchronization Errors $e_{1}, e_{2}, e_{3}, e_{4}$ 


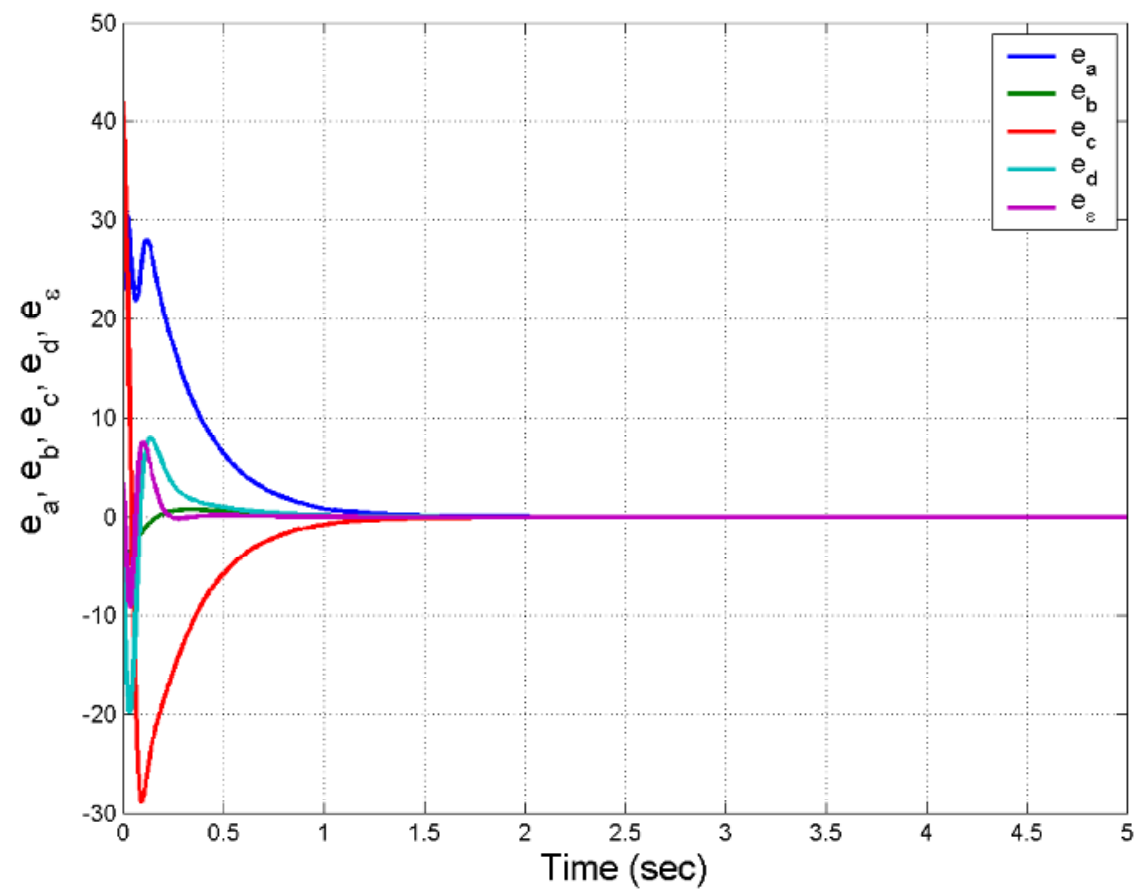

Figure 5. Time-History of the Parameter Estimation Errors $e_{a}, e_{b}, e_{c}, e_{d}, e_{\varepsilon}$

\section{Adaptive Control Design for the Anti-Synchronization of Hyperchaotic PAng Systems}

In this section, we design an adaptive controller for the anti-synchronization of two identical hyperchaotic Pang systems (2011) with unknown parameters.

Thedrive system is the hyperchaotic Pangdynamics given by

$$
\begin{aligned}
& \dot{x}_{1}=\alpha\left(x_{2}-x_{1}\right) \\
& \dot{x}_{2}=\gamma x_{2}-x_{1} x_{3}+x_{4} \\
& \dot{x}_{3}=-\beta x_{3}+x_{1} x_{2} \\
& \dot{x}_{4}=-\delta\left(x_{1}+x_{2}\right)
\end{aligned}
$$

where $\alpha, \beta, \gamma, \delta$ are unknown parameters of the system and $x \in R^{4}$ is the state.

The response system is the controlled hyperchaotic Pangdynamics given by

$$
\begin{aligned}
& \dot{y}_{1}=\alpha\left(y_{2}-y_{1}\right)+u_{1} \\
& \dot{y}_{2}=\gamma y_{2}-y_{1} y_{3}+y_{4}+u_{2} \\
& \dot{y}_{3}=-\beta y_{3}+y_{1} y_{2}+u_{3} \\
& \dot{y}_{4}=-\delta\left(y_{1}+y_{2}\right)+u_{4}
\end{aligned}
$$


where $y \in R^{4}$ is the state and $u_{1}, u_{2}, u_{3}, u_{4}$ are the adaptivecontrollers to be designed.

For the anti-synchronization, the error $e$ is defined as

$$
e_{1}=y_{1}+x_{1}, \quad e_{2}=y_{2}+x_{2}, \quad e_{3}=y_{3}+x_{3}, e_{4}=y_{4}+x_{4}
$$

Then we derive the error dynamics as

$$
\begin{aligned}
& \dot{e}_{1}=\alpha\left(e_{2}-e_{1}\right)+u_{1} \\
& \dot{e}_{2}=\gamma e_{2}+e_{4}-y_{1} y_{3}-x_{1} x_{3}+u_{2} \\
& \dot{e}_{3}=-\beta e_{3}+y_{1} y_{2}+x_{1} x_{2}+u_{3} \\
& \dot{e}_{4}=-\delta\left(e_{1}+e_{2}\right)+u_{4}
\end{aligned}
$$

The adaptive controller to achieve anti-synchronization is chosen as

$$
\begin{aligned}
& u_{1}=-\hat{\alpha}(t)\left(e_{2}-e_{1}\right)-k_{1} e_{1} \\
& u_{2}=-\hat{\gamma}(t) e_{2}-e_{4}+y_{1} y_{3}+x_{1} x_{3}-k_{2} e_{2} \\
& u_{3}=\hat{\beta}(t) e_{3}-y_{1} y_{2}-x_{1} x_{2}-k_{3} e_{3} \\
& u_{4}=\hat{\delta}(t)\left(e_{1}+e_{2}\right)-k_{4} e_{4}
\end{aligned}
$$

In Eq. (31), $k_{i},(i=1,2,3,4)$ are positive gains and $\hat{\alpha}(t), \hat{\beta}(t), \hat{\gamma}(t), \hat{\delta}(t)$ are estimates for the unknown parameters $\alpha, \beta, \gamma, \delta$ respectively.

By the substitution of (31) into (30), the error dynamics is simplified as

$$
\begin{aligned}
& \dot{e}_{1}=(\alpha-\hat{\alpha}(t))\left(e_{2}-e_{1}\right)-k_{1} e_{1} \\
& \dot{e}_{2}=(\gamma-\hat{\gamma}(t)) e_{2}-k_{2} e_{2} \\
& \dot{e}_{3}=-(\beta-\hat{\beta}(t)) e_{3}-k_{3} e_{3} \\
& \dot{e}_{4}=-(\delta-\hat{\delta}(t))\left(e_{1}+e_{2}\right)-k_{4} e_{4}
\end{aligned}
$$

As a next step, we define the parameter estimation errors as

$$
e_{\alpha}(t)=\alpha-\hat{\alpha}(t), e_{\beta}(t)=\beta-\hat{\beta}(t), e_{\gamma}(t)=\gamma-\hat{\gamma}(t), e_{\delta}(t)=\delta-\hat{\delta}(t)
$$

Upon differentiation, we get

$$
\dot{e}_{\alpha}(t)=-\dot{\hat{\alpha}}(t), \dot{e}_{\beta}(t)=-\dot{\hat{\beta}}(t), \dot{e}_{\gamma}(t)=-\dot{\hat{\gamma}}(t), \dot{e}_{\delta}(t)=-\dot{\hat{\delta}}(t)
$$

Substituting (33) into the error dynamics (32), we obtain 


$$
\begin{aligned}
& \dot{e}_{1}=e_{\alpha}\left(e_{2}-e_{1}\right)-k_{1} e_{1} \\
& \dot{e}_{2}=e_{\gamma} e_{2}-k_{2} e_{2} \\
& \dot{e}_{3}=-e_{\beta} e_{3}-k_{3} e_{3} \\
& \dot{e}_{4}=-e_{\delta}\left(e_{1}+e_{2}\right)-k_{4} e_{4}
\end{aligned}
$$

We consider the candidate Lyapunov function

$$
V=\frac{1}{2}\left(e_{1}^{2}+e_{2}^{2}+e_{3}^{2}+e_{4}^{2}+e_{\alpha}^{2}+e_{\beta}^{2}+e_{\gamma}^{2}+e_{\delta}^{2}\right)
$$

Differentiating (36) along the dynamics (34) and (35), we obtain

$$
\begin{aligned}
\dot{V}= & -k_{1} e_{1}^{2}-k_{2} e_{2}^{2}-k_{3} e_{3}^{2}-k_{4} e_{4}^{2}+e_{\alpha}\left[e_{1}\left(e_{2}-e_{1}\right)-\dot{\hat{\alpha}}\right]+e_{\beta}\left(-e_{3}^{2}-\dot{\hat{\beta}}\right) \\
& +e_{\gamma}\left(e_{2}^{2}-\dot{\hat{\gamma}}\right)+e_{\delta}\left(-e_{1} e_{4}\left(e_{1}+e_{2}\right)-\dot{\hat{\delta}}\right)
\end{aligned}
$$

In view of (37), we choose the following parameter update law:

$$
\begin{aligned}
& \dot{\hat{\alpha}}=e_{1}\left(e_{2}-e_{1}\right)+k_{5} e_{\alpha} \\
& \dot{\hat{\beta}}=-e_{3}^{2}+k_{6} e_{\beta} \\
& \dot{\hat{\gamma}}=e_{2}^{2}+k_{7} e_{\gamma} \\
& \dot{\hat{\delta}}=-e_{1} e_{4}\left(e_{1}+e_{2}\right)+k_{8} e_{\delta}
\end{aligned}
$$

Next, we prove the following main result of this section.

Theorem 5.1 The adaptive control law defined by Eq. (31) along with the parameter update law defined by Eq. (38) achieve global and exponential anti-synchronization of the identical hyperchaotic Pang systems (27) and (28) with unknown parameters for all initial conditions $x(0), y(0) \in R^{4}$. Moreover, the parameter estimation errors $e_{\alpha}(t), e_{\beta}(t), e_{\gamma}(t), e_{\delta}(t)$ globally and exponentially converge to zero for all initial conditions.

Proof.The proof is via Lyapunov stability theory [26] by taking $V$ defined by Eq. (36) as the candidate Lyapunov function. Substituting the parameter update law (38) into (37), we get

$$
\dot{V}(e)=-k_{1} e_{1}^{2}-k_{2} e_{2}^{2}-k_{3} e_{3}^{2}-k_{4} e_{4}^{2}-k_{5} e_{a}^{2}-k_{6} e_{b}^{2}-k_{7} e_{c}^{2}-k_{8} e_{d}^{2}-k_{9} e_{\varepsilon}^{2}
$$

which is a negative definite function on $R^{9}$. This completes the proof.

Next, we illustrate our adaptive anti-synchronization results with MATLAB simulations. The classical fourth order Runge-Kutta method with time-step $h=10^{-8}$ has been used to solve the hyperchaotic Pang systems (27) and (28) with the nonlinear controller defined by (31).

The feedback gains in the adaptive controller (31) are taken as $k_{i}=4,(i=1, \ldots, 8)$. 
International Journal of Instrumentation and Control Systems (IJICS) Vol.3, No.2, April 2013

The parameters of the hyperchaotic Pang systems are taken as in the hyperchaotic case, i.e.

$$
\alpha=36, \quad \beta=3, \quad \gamma=20, \quad \delta=2
$$

For simulations, the initial conditions of the drive system (27) are taken as

$$
x_{1}(0)=17, \quad x_{2}(0)=-22, \quad x_{3}(0)=-11, x_{4}(0)=25
$$

Also, the initial conditions of the response system (28) are taken as

$$
y_{1}(0)=24, \quad y_{2}(0)=-18, \quad y_{3}(0)=24, \quad y_{4}(0)=-17
$$

Also, the initial conditions of the parameter estimates are taken as

$$
\hat{\alpha}(0)=3, \hat{\beta}(0)=-4, \quad \hat{\gamma}(0)=27, \hat{\delta}(0)=15
$$

Figure 6depicts the anti-synchronization of the identical hyperchaotic Pang systems.

Figure 7depicts the time-history of the anti-synchronization errors $e_{1}, e_{2}, e_{3}, e_{4}$.

Figure 8 depicts the time-history of the parameter estimation errors $e_{\alpha}, e_{\beta}, e_{\gamma}, e_{\delta}$.
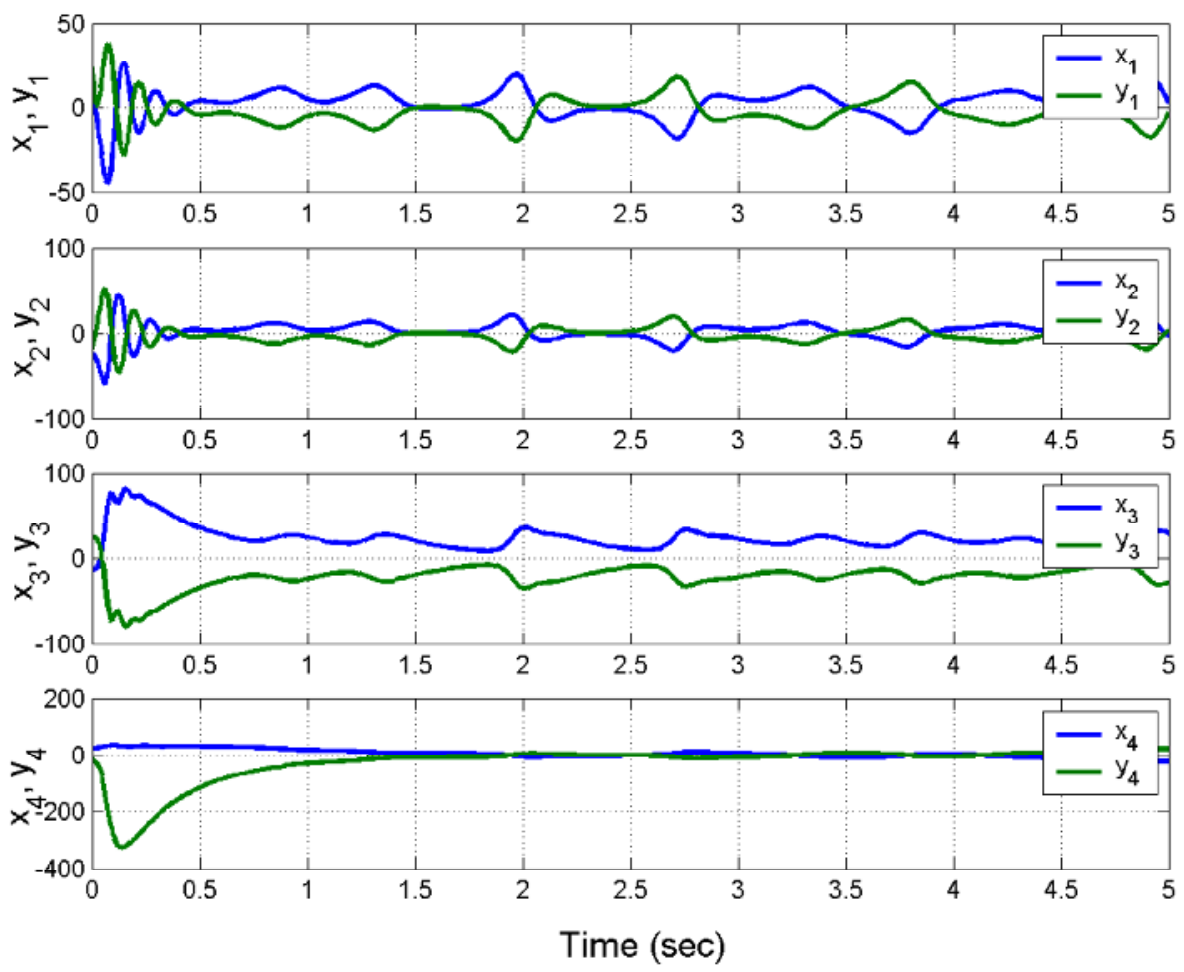

Figure 6. Anti-Synchronization of Identical Hyperchaotic Pang Systems 
International Journal of Instrumentation and Control Systems (IJICS) Vol.3, No.2, April 2013

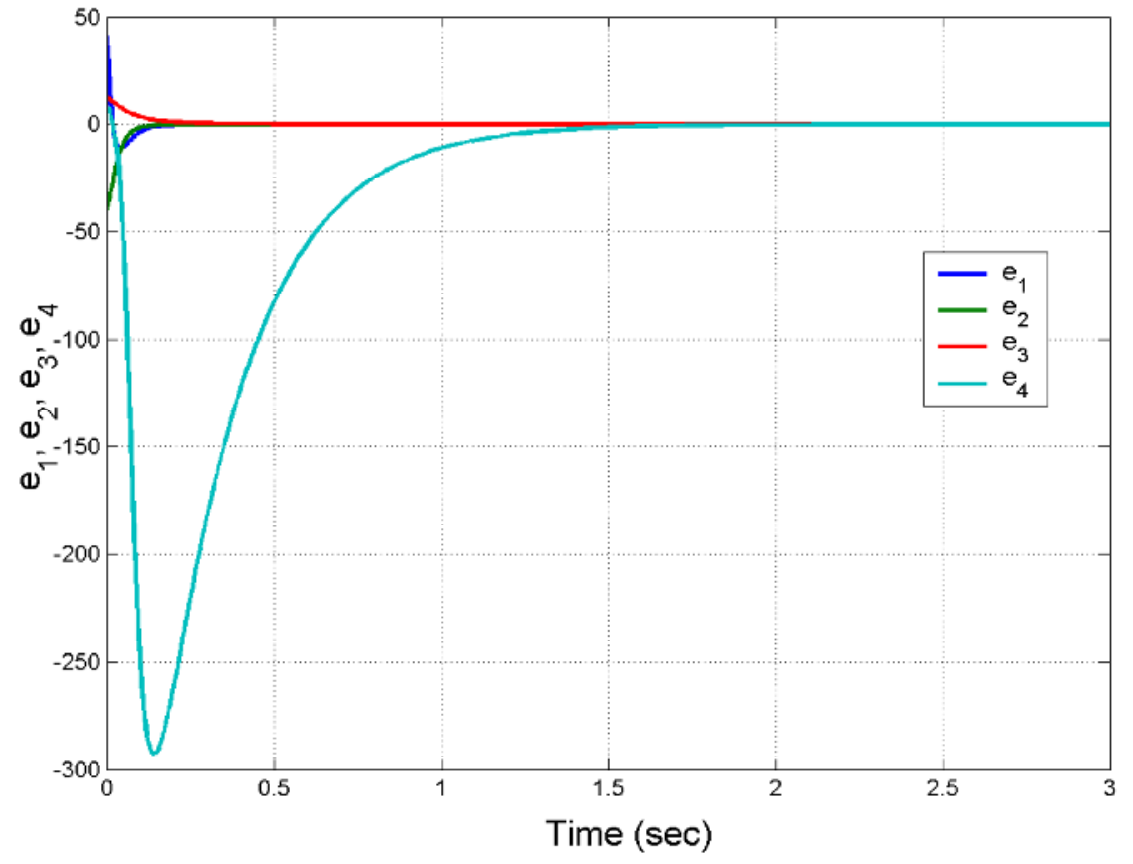

Figure 7. Time-History of the Anti-Synchronization Errors $e_{1}, e_{2}, e_{3}, e_{4}$

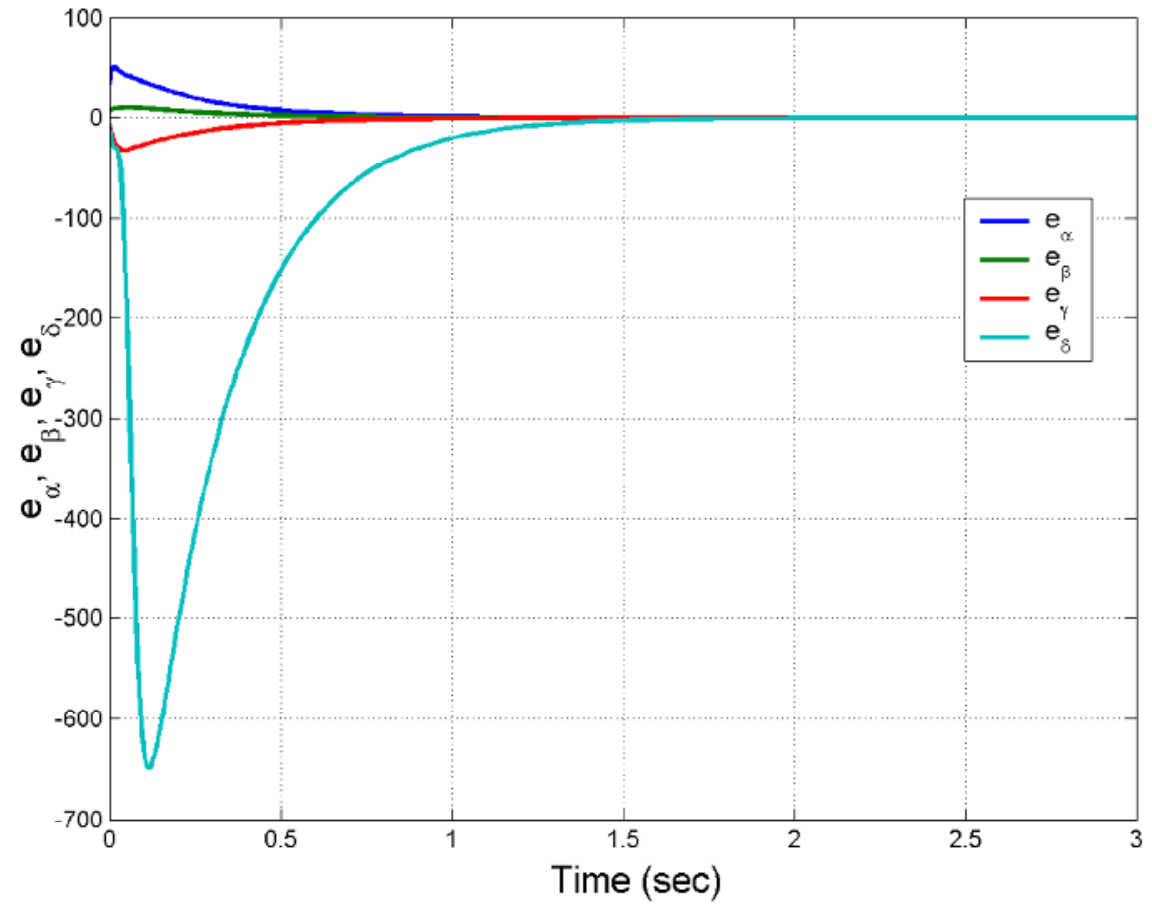

Figure 8. Time-History of the Parameter Estimation Errors $e_{\alpha}, e_{\beta}, e_{\gamma}, e_{\delta}$ 


\section{AdAPtive Control Design FOR the Anti-SynChronization of HyPERCHAOTIC YANG AND HyPERCHAOTIC PANG SYSTEMS}

In this section, we design an adaptive controller for the anti-synchronization of non-identical hyperchaotic Yang (2009) and hyperchaotic Pang systems (2011) with unknown parameters. Thedrive system is the hyperchaotic Yangdynamics given by

$$
\begin{aligned}
& \dot{x}_{1}=a\left(x_{2}-x_{1}\right) \\
& \dot{x}_{2}=c x_{1}-x_{1} x_{3}+x_{4} \\
& \dot{x}_{3}=-b x_{3}+x_{1} x_{2} \\
& \dot{x}_{4}=-d x_{1}-\varepsilon x_{2}
\end{aligned}
$$

where $a, b, c, d, \varepsilon$ are unknown parameters of the system and $x \in R^{4}$ is the state.

The response system is the controlled hyperchaotic Pangdynamics given by

$$
\begin{aligned}
& \dot{y}_{1}=\alpha\left(y_{2}-y_{1}\right)+u_{1} \\
& \dot{y}_{2}=\gamma y_{2}-y_{1} y_{3}+y_{4}+u_{2} \\
& \dot{y}_{3}=-\beta y_{3}+y_{1} y_{2}+u_{3} \\
& \dot{y}_{4}=-\delta\left(y_{1}+y_{2}\right)+u_{4}
\end{aligned}
$$

where $\alpha, \beta, \gamma, \delta$ are unknown parameters, $y \in R^{4}$ is the state and $u_{1}, u_{2}, u_{3}, u_{4}$ are the adaptivecontrollers to be designed.

For the anti-synchronization, the error $e$ is defined as

$$
e_{1}=y_{1}+x_{1}, \quad e_{2}=y_{2}+x_{2}, \quad e_{3}=y_{3}+x_{3}, \quad e_{4}=y_{4}+x_{4}
$$

Then we derive the error dynamics as

$$
\begin{aligned}
& \dot{e}_{1}=\alpha\left(y_{2}-y_{1}\right)+a\left(x_{2}-x_{1}\right)+u_{1} \\
& \dot{e}_{2}=\gamma y_{2}+e_{4}+c x_{1}-y_{1} y_{3}-x_{1} x_{3}+u_{2} \\
& \dot{e}_{3}=-\beta y_{3}-b x_{3}+y_{1} y_{2}+x_{1} x_{2}+u_{3} \\
& \dot{e}_{4}=-\delta\left(y_{1}+y_{2}\right)-d x_{1}-\varepsilon x_{2}+u_{4}
\end{aligned}
$$

The adaptive controller to achieve anti-synchronization is chosen as

$$
\begin{aligned}
& u_{1}=-\hat{\alpha}(t)\left(y_{2}-y_{1}\right)-\hat{a}(t)\left(x_{2}-x_{1}\right)-k_{1} e_{1} \\
& u_{2}=-\hat{\gamma}(t) y_{2}-e_{4}-\hat{c}(t) x_{1}+y_{1} y_{3}+x_{1} x_{3}-k_{2} e_{2} \\
& u_{3}=\hat{\beta}(t) y_{3}+\hat{b}(t) x_{3}-y_{1} y_{2}-x_{1} x_{2}-k_{3} e_{3} \\
& u_{4}=\hat{\delta}(t)\left(y_{1}+y_{2}\right)+\hat{d}(t) x_{1}+\hat{\varepsilon}(t) x_{2}-k_{4} e_{4}
\end{aligned}
$$


In Eq. (44), $k_{i},(i=1,2,3,4)$ are positive gains, $\hat{a}(t), \hat{b}(t), \hat{c}(t), \hat{d}(t), \hat{\varepsilon}(t)$ are estimates for the unknown parameters $a, b, c, d, \varepsilon$ respectively, and $\hat{\alpha}(t), \hat{\beta}(t), \hat{\gamma}(t), \hat{\delta}(t)$ are estimates for the unknown parameters $\alpha, \beta, \gamma, \delta$ respectively.

By the substitution of (44) into (43), the error dynamics is simplified as

$$
\begin{aligned}
& \dot{e}_{1}=(\alpha-\hat{\alpha}(t))\left(y_{2}-y_{1}\right)+(a-\hat{a}(t))\left(x_{2}-x_{1}\right)-k_{1} e_{1} \\
& \dot{e}_{2}=(\gamma-\hat{\gamma}(t)) y_{2}+(c-\hat{c}(t)) x_{1}-k_{2} e_{2} \\
& \dot{e}_{3}=-(\beta-\hat{\beta}(t)) y_{3}-(b-\hat{b}(t)) x_{3}-k_{3} e_{3} \\
& \dot{e}_{4}=-(\delta-\hat{\delta}(t))\left(y_{1}+y_{2}\right)-(d-\hat{d}(t)) x_{1}-(\varepsilon-\hat{\varepsilon}(t)) x_{2}-k_{4} e_{4}
\end{aligned}
$$

As a next step, we define the parameter estimation errors as

$$
\begin{aligned}
& e_{a}(t)=a-\hat{a}(t), e_{b}(t)=b-\hat{b}(t), e_{c}(t)=c-\hat{c}(t), e_{d}(t)=d-\hat{d}(t), e_{\varepsilon}(t)=\varepsilon-\hat{\varepsilon}(t) \\
& e_{\alpha}(t)=\alpha-\hat{\alpha}(t), e_{\beta}(t)=\beta-\hat{\beta}(t), e_{\gamma}(t)=\gamma-\hat{\gamma}(t), e_{\delta}(t)=\delta-\hat{\delta}(t)
\end{aligned}
$$

Upon differentiation, we get

$$
\begin{aligned}
& \dot{e}_{a}(t)=-\dot{\hat{a}}(t), \dot{e}_{b}(t)=-\dot{\hat{b}}(t), \dot{e}_{c}(t)=-\dot{\hat{c}}(t), \dot{e}_{d}(t)=-\dot{\hat{d}}(t), \dot{e}_{\varepsilon}(t)=-\dot{\hat{\varepsilon}}(t) \\
& \dot{e}_{\alpha}(t)=-\dot{\hat{\alpha}}(t), \dot{e}_{\beta}(t)=-\dot{\hat{\beta}}(t), \dot{e}_{\gamma}(t)=-\dot{\hat{\gamma}}(t), \dot{e}_{\delta}(t)=-\dot{\hat{\delta}}(t)
\end{aligned}
$$

Substituting (46) into the error dynamics (45), we obtain

$$
\begin{aligned}
& \dot{e}_{1}=e_{\alpha}\left(y_{2}-y_{1}\right)+e_{a}\left(x_{2}-x_{1}\right)-k_{1} e_{1} \\
& \dot{e}_{2}=e_{\gamma} y_{2}+e_{c} x_{1}-k_{2} e_{2} \\
& \dot{e}_{3}=-e_{\beta} y_{3}-e_{b} x_{3}-k_{3} e_{3} \\
& \dot{e}_{4}=-e_{\delta}\left(y_{1}+y_{2}\right)-e_{d} x_{1}-e_{\varepsilon} x_{2}-k_{4} e_{4}
\end{aligned}
$$

We consider the candidate Lyapunov function

$$
V=\frac{1}{2}\left(e_{1}^{2}+e_{2}^{2}+e_{3}^{2}+e_{4}^{2}+e_{a}^{2}+e_{b}^{2}+e_{c}^{2}+e_{d}^{2}+e_{\varepsilon}^{2}+e_{\alpha}^{2}+e_{\beta}^{2}+e_{\gamma}^{2}+e_{\delta}^{2}\right)
$$

Differentiating (49) along the dynamics (47) and (48), we obtain

$$
\begin{aligned}
\dot{V}= & -k_{1} e_{1}^{2}-k_{2} e_{2}^{2}-k_{3} e_{3}^{2}-k_{4} e_{4}^{2}+e_{a}\left[e_{1}\left(x_{2}-x_{1}\right)-\dot{\hat{a}}\right]+e_{b}\left[-e_{3} x_{3}-\dot{\hat{b}}\right]+e_{c}\left[e_{2} x_{1}-\dot{\hat{c}}\right] \\
& +e_{d}\left[-e_{4} x_{1}-\dot{\hat{d}}\right]+e_{\varepsilon}\left[-e_{4} x_{2}-\dot{\hat{\varepsilon}}\right]+e_{\alpha}\left[e_{1}\left(y_{2}-y_{1}\right)-\dot{\hat{\alpha}}\right]+e_{\beta}\left[-e_{3} y_{3}-\dot{\hat{\beta}}\right] \\
& +e_{\gamma}\left[e_{2} y_{2}-\dot{\hat{\gamma}}\right]+e_{\delta}\left[-e_{4}\left(y_{1}+y_{2}\right)-\dot{\hat{\delta}}\right]
\end{aligned}
$$


In view of (50), we choose the following parameter update law:

$$
\begin{array}{ll}
\dot{\hat{a}}=e_{1}\left(x_{2}-x_{1}\right)+k_{5} e_{a}, & \dot{\hat{\alpha}}=e_{1}\left(y_{2}-y_{1}\right)+k_{10} e_{\alpha} \\
\dot{\hat{b}}=-e_{3} x_{3}+k_{6} e_{b}, & \dot{\hat{\beta}}=-e_{3} y_{3}+k_{11} e_{\beta} \\
\dot{\hat{c}}=e_{2} x_{1}+k_{7} e_{c}, & \dot{\hat{\gamma}}=e_{2} y_{2}+k_{12} e_{\gamma} \\
\dot{\hat{d}}=-e_{4} x_{1}+k_{8} e_{d}, & \dot{\hat{\delta}}=-e_{4}\left(y_{1}+y_{2}\right)+k_{13} e_{\delta} \\
\dot{\hat{\varepsilon}}=-e_{4} x_{2}+k_{9} e_{\varepsilon} &
\end{array}
$$

Theorem 6.1 The adaptive control law defined by Eq. (44) along with the parameter update law defined by Eq. (51) achieve global and exponential anti-synchronization of the non-identical hyperchaotic Yang system (40) and hyperchaotic Pang system (41) with unknown parameters for all initial conditions $x(0), y(0) \in R^{4}$. Moreover, all the parameter estimation errors globally and exponentially converge to zero for all initial conditions.

Proof.The proof is via Lyapunov stability theory [26] by taking $V$ defined by Eq. (49) as the candidate Lyapunov function. Substituting the parameter update law (51) into (50), we get

$$
\begin{aligned}
\dot{V}(e)= & -k_{1} e_{1}^{2}-k_{2} e_{2}^{2}-k_{3} e_{3}^{2}-k_{4} e_{4}^{2}-k_{5} e_{a}^{2}-k_{6} e_{b}^{2}-k_{7} e_{c}^{2}-k_{8} e_{d}^{2}-k_{9} e_{\varepsilon}^{2} \\
& -k_{10} e_{\alpha}^{2}-k_{11} e_{\beta}^{2}-k_{12} e_{\gamma}^{2}-k_{13} e_{\delta}^{2}
\end{aligned}
$$

which is a negative definite function on $R^{13}$. This completes the proof.

Next, we illustrate our adaptive anti-synchronization results with MATLAB simulations. The classical fourth order Runge-Kutta method with time-step $h=10^{-8}$ has been used to solve the hyperchaotic systems (40) and (41) with the nonlinear controller defined by (44). The feedback gains in the adaptive controller (31) are taken as $k_{i}=4,(i=1, \ldots, 8)$.

The parameters of the two hyperchaoticsystems are taken as in the hyperchaotic case, i.e.

$$
a=35, \quad b=3, \quad c=35, \quad d=2, \quad \varepsilon=7.5, \quad \alpha=36, \quad \beta=3, \quad \gamma=20, \quad \delta=2
$$

For simulations, the initial conditions of the drive system (40) are taken as

$$
x_{1}(0)=29, \quad x_{2}(0)=14, \quad x_{3}(0)=-23, \quad x_{4}(0)=-9
$$

Also, the initial conditions of the response system (41) are taken as

$$
y_{1}(0)=14, \quad y_{2}(0)=-18, \quad y_{3}(0)=29, \quad y_{4}(0)=-14
$$

Also, the initial conditions of the parameter estimates are taken as 
International Journal of Instrumentation and Control Systems (IJICS) Vol.3, No.2, April 2013

$$
\begin{aligned}
& \hat{a}(0)=9, \hat{b}(0)=4, \hat{c}(0)=-3, \hat{d}(0)=8, \hat{\varepsilon}(0)=-4, \\
& \hat{\alpha}(0)=6, \hat{\beta}(0)=2, \hat{\gamma}(0)=11, \hat{\delta}(0)=-9
\end{aligned}
$$

Figure 9depicts the anti-synchronization of the non-identical hyperchaotic Yang and hyperchaotic Pang systems. Figure 10depicts the time-history of the anti-synchronization errors $e_{1}, e_{2}, e_{3}, e_{4}$. Figure 11 depicts the time-history of the parameter estimation errors $e_{a}, e_{b}, e_{c}, e_{d}, \mathrm{e}_{\varepsilon}$. Figure 12 depicts the time-history of the parameter estimation errors $e_{\alpha}, e_{\beta}, e_{\gamma}, e_{\delta}$.
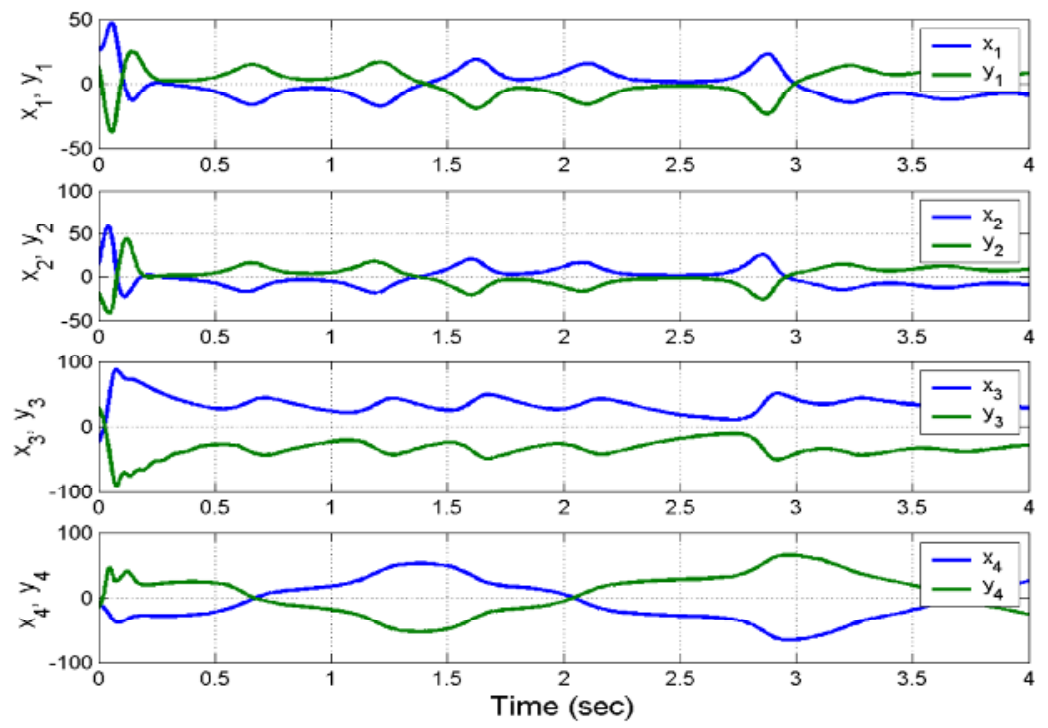

Figure 9. Anti-Synchronization of Hyperchaotic Yang and Hyperchaotic Pang Systems

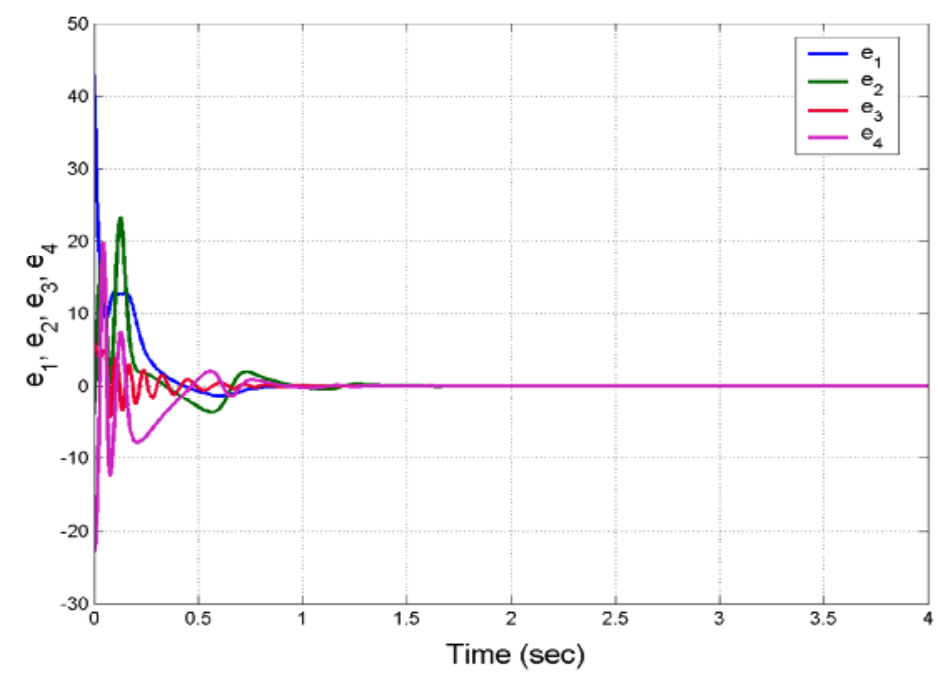

Figure 10. Time-History of the Anti-Synchronization Errors $e_{1}, e_{2}, e_{3}, e_{4}$ 
International Journal of Instrumentation and Control Systems (IJICS) Vol.3, No.2, April 2013

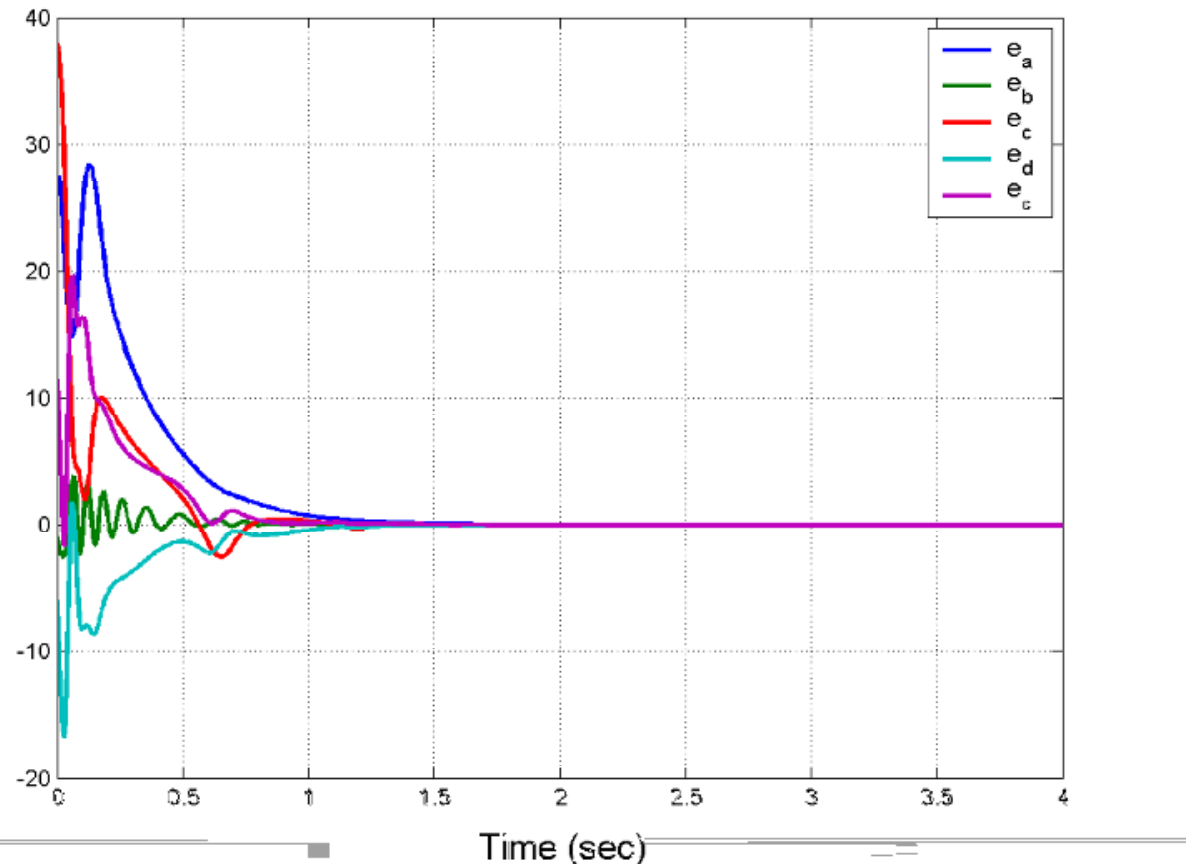

Figure 11. Time-History of the Parameter Estimation Errors $e_{a}, e_{b}, e_{c}, e_{d}, e_{\varepsilon}$

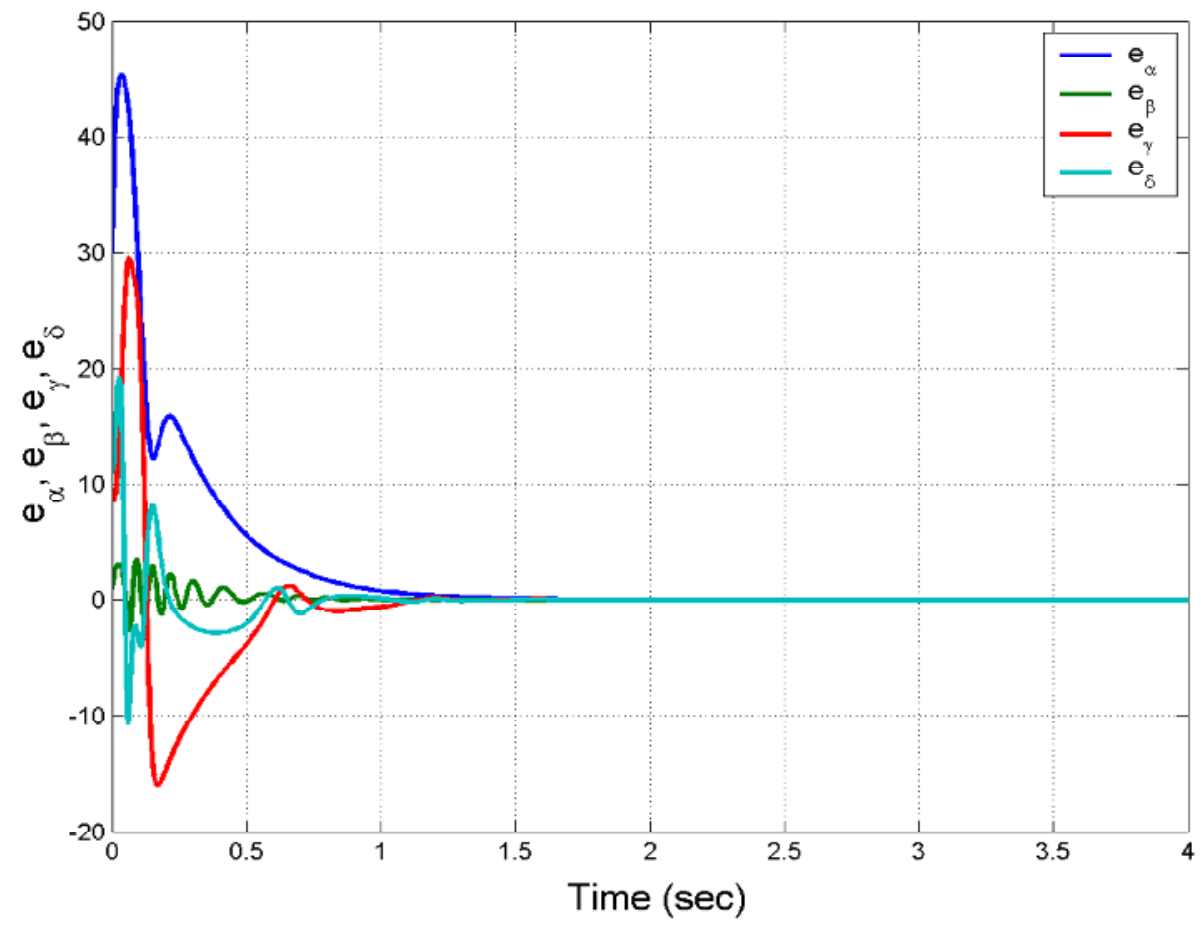

Figure 12. Time-History of the Parameter Estimation Errors $e_{\alpha}, e_{\beta}, e_{\gamma}, e_{\delta}$ 
International Journal of Instrumentation and Control Systems (IJICS) Vol.3, No.2, April 2013

\section{CONCLUSIONS}

In this paper, we have used adaptive control to derive new results for the anti-synchronization of hyperchaotic Yang system (2009) and hyperchaotic Pang system (2011) with unknown parameters. Main results of anti-synchronization design results for hyperchaotic systems addressed in this paper were proved using adaptive control theory and Lyapunov stability theory. Hyperchaotic systems have important applications in areas like secure communication, data encryption, neural networks, etc.MATLAB simulations have been shown to validate and demonstrate the adaptive anti-synchronization results for hyperchaotic Yang and hyperchaotic Pang systems.

\section{REFERENCES}

[1] Rössler, O.E. (1979) “An equation for hyperchaos,” Physics Letters A, Vol. 71, pp 155-157.

[2] Machado, L.G., Savi, M.A. \& Pacheco, P.M.C.L. (2003) "Nonlinear dynamics and chaos in coupled shape memory oscillators," International Journal of Solids and Structures, Vol. 40, No. 19, pp. 5139-5156.

[3] Huang, Y. \& Yang, X.S. (2006) "Hyperchaos and bifurcation in a new class of four-dimensional Hopfield neural networks," Neurocomputing, Vol. 69, pp 13-15.

[4] Tao, Y. (1999) "Chaotic secure communication systems - history and new results", Telecommun. Review, Vol. 9, pp 597-634.

[5] Li, C., Liao, X. \& Wong, K.W. (2005) "Lag synchronization of hyperchaos with applications to secure communications," Chaos, Solitons \& Fractals, Vol. 23, No. 1, pp 183-193.

[6] Prokhorov, M.D. \& Ponomarenko, V.I. (2008) "Encryption and decryption of information in chaotic communication systems governed by delay-differential equations," Chaos, Solitons \& Fractals, Vol. 35, No. 5, pp 871-877.

[7] Yassen, M.T. (2008) "Synchronization hyperchaos of hyperchaotic systems", Chaos, Solitons and Fractals, Vol. 37, pp 465-475.

[8] Pecora, L.M. \& Carroll, T.L. (1990) "Synchronization in chaotic systems", Phys. Rev. Lett., Vol. 64, pp 821-824.

[9] Ott, E., Grebogi, C. \& Yorke, J.A. (1990) “Controlling chaos”, Phys. Rev. Lett., Vol. 64, pp 11961199.

[10] Huang, L. Feng, R. \& Wang, M. (2004) "Synchronization of chaotic systems via nonlinear control," Physics Letters A, Vol. 320, No. 4, pp 271-275.

[11] Lei, Y., Xu, W. \& Zheng, H. (2005) "Synchronization of two chaotic nonlinear gyros using active control," Physics Letters A, Vol. 343, pp 153-158.

[12] Sarasu, P. \& Sundarapandian, V. (2011) "Active controller design for generalized projective synchronization of four-scroll chaotic systems", International Journal of System Signal Control and Engineering Application, Vol. 4, No. 2, pp 26-33.

[13] Sarasu, P. \& Sundarapandian, V. (2012) "Generalized projective synchronization of two-scroll systems via adaptive control," International Journal of Soft Computing, Vol. 7, No. 4, pp 146-156.

[14] Sundarapandian, V. (2012) "Adaptive control and synchronization of a generalized Lotka-Volterra system," Vol. 1, No. 1, pp 1-12.

[15] Sundarapandian, V. (2013) "Adaptive controller and synchronizer design for hyperchaotic Zhou system with unknown parameters," Vol. 1, No. 1, pp 18-32.

[16] Bowong, S. \&Kakmeni, F.M.M. (2004) "Synchronization of uncertain chaotic systems via backstepping approach,” Chaos, Solitons \& Fractals, Vol. 21, No. 4, pp 999-1011.

[17] Suresh, R, \& Sundarapandian, V. (2012) "Global chaos synchronization of WINDMI and Coullet chaotic systems by backstepping control”, Far East J. Math. Sciences, Vol. 67, No. 2, pp 265-287.

[18] Suresh, R. \& Sundarapandian, V. (2012) "Hybrid synchronization of n-scroll Chua and Lur'e chaotic systems via backstepping control with novel feedback", Arch. Control Sciences, Vol. 22, No. 3, pp 255-278. 
International Journal of Instrumentation and Control Systems (IJICS) Vol.3, No.2, April 2013

[19] Sundarapandian, V. (2013) "Anti-synchronizing backstepping design for Arneodo chaotic system”, International Journal on Bioinformatics and Biosciences, Vol. 3, No. 1, pp 21-33.

[20] Senejohnny, D.M. \& Delavari, H. (2012) "Active sliding observer scheme based fractional chaos synchronization," Comm. Nonlinear Sci. Numerical Simulation, Vol. 17, No. 11, pp 4373-4383.

[21] Sundarapandian, V. (2012) "Anti-synchronization of hyperchaotic Xu systems via sliding mode control”, International Journal of Embedded Systems, Vol. 2, No. 2, pp 51-61.

[22] Sundarapandian, V. (2013) "Anti-synchronizing sliding controller design for identical Pan systems," International Journal of Computational Science and Information Technology, Vol. 1, No. 1, pp 1-9.

[23] Yang, Q. \& Liu, Y. (2009) “A hyperchaotic system from a chaotic system with one saddle and two stable node-foci," J. Mathematical Analysis and Applications, Vol. 360, pp 293-306.

[24] Pang, S. \& Liu, Y. (2011) "A new hyperchaotic system from the Lü system and its control," Journal of Computational and Applied Mathematics, Vol. 235, pp 2775-2789.

[25] Aström, K.J. \& Wittenmark, B. (1994) Adaptive Control, $2^{\text {nd }}$ edition, Prentice Hall, N.Y., USA.

[26] Hahn, W. (1967) The Stability of Motion, Springer, Berlin.

\section{Author}

Dr. V. Sundarapandian earned his D.Sc. in Electrical and Systems Engineering from Washington University, St. Louis, USA in May 1996. He is Professor and Dean of the R \& D Centre at Vel Tech Dr. RR \& Dr. SR Technical University, Chennai, Tamil Nadu, India. So far, he has published over 300 research works in refereed international journals. He has also published over 200 research papers in National and International Conferences. He has delivered Key Note Addresses at many International Conferences with IEEE and Springer Proceedings. He is an India Chair of AIRCC. He is the Editor-in-Chief of the AIRCC Control Journals International Journal of Instrumentation and Control Systems, International Journal

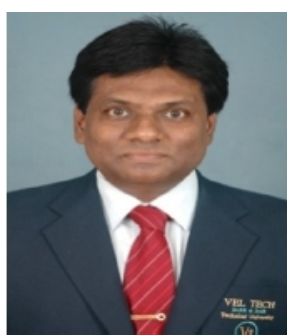
of Control Theory and Computer Modeling,International Journal of Information Technology, Control and Automation, International Journal of Chaos, Control, Modelling and Simulation, and International Journal of Information Technology, Modeling and Computing. His research interests are Control Systems, Chaos Theory, Soft Computing, Operations Research, Mathematical Modelling and Scientific Computing. He has published four text-books and conducted many workshops on Scientific Computing, MATLAB and SCILAB. 